\title{
Does aspirin therapy affect outcomes of patients underwent radical cystectomy?
}

\section{Hernández L, Castellarnau S, Sierra P, Sabaté S, Hernando D. \\ Fundacion Puigvert, Department of Anaesthesiology \& Pain Medicine, Barcelona, Spain}

Fundació Puigvert

\section{BACKGROUND AND GOAL OF STUDY:}

A clinical dilemma surrounds the use of aspirin therapy during radical cistectomy. The impact to interrupt or continue therapy with aspirin in the perioperative period have been recently studied with controversial results.

The current practice at our institution has been to continue aspirin therapy up to surgery.

The aim of this study was to assess safety of radical cistectomy in patients who received aspirin up to the day of surgery.

\section{MATERIAL AND METHODS:}

A total of 352 consecutive cases of radical cystectomy (open and laparoscopic) and urinary diversion between January 2011 and June 2015 in our centre. Perioperative data from patients who underwent radical cystectomy were compiled and analyzed according to the use of aspirin.

The unadjusted and adjusted relationship between aspirin use and postoperative complications was evaluated using logistic regression analysis and by the propensity score for aspirin use.

\section{RESULTS:}

We included 352 patients, 91 (25.9\%) received aspirin up to the day of surgery. Demographic data and preoperative risk factors are shown in table 1. Inta and postoperative data in table 2 . Table 3 shows the unadjusted and adjusted in-hospital complications.

Table 1. Demographic data and preoperative risk factors

\begin{tabular}{|l|c|c|c|}
\multicolumn{1}{c}{} & \multicolumn{1}{c}{ Aspirin } & No aspirin & P \\
\hline N & $91(25.9)$ & $261(74.1)$ & \\
\hline Age ** & $68.5(67.2-69.8)$ & $71.9(70.2-73.6)$ & 0.002 \\
\hline Gender (M/F) (n,\%) & $89(97.8) / 2(2.2)$ & $238(91.2) / 23(8.8)$ & 0.034 \\
\hline BMI ** & $27.4(26.2-28.5)$ & $27.2(26.6-27.7)$ & 0.693 \\
\hline ASA physical status (1/I/III/IV) (\%) & $0 / 20,9 / 67 / 12.1$ & $7.7 / 50.2 / 37.9 / 4.2$ & $<0.001$ \\
\hline Hypertension (n,\%) & $61(67.0)$ & $121(46.4)$ & $<0.001$ \\
\hline Coronary artery disease (n,\%) & $23(25.3)$ & $1(0.4)$ & $<0.001$ \\
\hline Chronic heart failure (n,\%) & $12(13.2)$ & $8(3.1)$ & 0.001 \\
\hline COPD (n,\%) & $26(28.6)$ & $62(23.8)$ & 0.473 \\
\hline Smokers (n,\%) & $63(69.2)$ & $172(65.9)$ & 0.248 \\
\hline Diabetes (n,\%) & $29(31.8)$ & $43(16.5)$ & 0.001 \\
\hline Dislipidaemia (n,\%) & $54(59.3)$ & $74(28.4)$ & $<0.001$ \\
\hline Peripheral artery disease (n,\%) & $30(32.9)$ & $19(7.3)$ & $<0.001$ \\
\hline Cerebrovascular disease (n,\%) & $10(10.9)$ & $8(3.1)$ & 0.005 \\
\hline Chronic kidney disease (n,\%) & $29(31.9)$ & $44(16.9)$ & 0.003 \\
\hline
\end{tabular}

*Median (25-75th percentile); ** Mean $(95 \% \mathrm{Cl})$

Aspirin patients had more co-morbidity and worse functional class
Table 2. Unadjusted intra-postoperative data

$$
\text { Aspirin }
$$

No aspirin $\quad P$

\begin{tabular}{|l|c|c|c|}
\hline Surgery (open/laparoscopic) (n,\%) & $70(76.9) / 21(23.1)$ & $162(62.1) / 99(37.9)$ & 0.01 \\
\hline Anaesthesia (general/combined) (n,\%) & $14(15.4) / 77(84.6)$ & $41(15.7) / 220(84.3)$ & 0.942 \\
\hline Duration of procedure (min)* & $325(270-390)$ & $350(275-410)$ & 0.111 \\
\hline Fluids (ml/kg/h)* & $8.5(6.2-11.9)$ & $8.1(6.3-10.4)$ & 0.569 \\
\hline Col.loid (ml) & $51(56.0)$ & $145(55.5)$ & 0.878 \\
\hline Estimated blood loss (ml.)* & $500(400-1200)$ & $500(300-750)$ & 0.027 \\
\hline Hb (g/L) before surgery ** & $125.6(121.6-129.7)$ & $133.3(130.9-135.6)$ & 0.001 \\
\hline Hb (g/L) hospital discharge** & $103.9(101.3-106.6)$ & $108.9(107.2-110.7)$ & 0.003 \\
\hline Red blood cell transfusion (n,\%) & $49(53.8)$ & $88(33.7)$ & $<0.001$ \\
\hline Recovery stay (h)* & $19.8(17.3-22.5)$ & $18.5(15.5-20.8)$ & 0.058 \\
\hline
\end{tabular}

*Median (25-75th percentile); ${ }^{* *}$ Mean $(95 \% \mathrm{Cl})$

Unadjusted intraoperative blood loss, red blood cell transfusion, were significantly higher in aspirin group

Table 3. In-hospital postoperative events related to aspirin use Unadjusted OR (95\% Cl)

$\mathbf{P}$

\begin{tabular}{|l|c|c|c|}
\hline Complications (any) & $1.2(0.7-1.9)$ & 0.516 & $1.1(0.5-2.2)$ \\
\hline RBC transfusion & $2.5(1.5-4.1)$ & $<0.001$ & $1.2(0.6-2.4)$ \\
\hline Postoperative blood loss $(\mathbf{m l ) *}$ & $146.1(35.4-256.8)$ & 0.010 & $85.9(-68.8-240.7)$ \\
\hline Respiratory & $2.1(0.9-4.4)$ & 0.051 & $2.2(0.7-6.3)$ \\
\hline Cardiac & $2.9(1.1-7.3)$ & 0.027 & $1.7(0.4-6.8)$ \\
\hline Neurologic & $2.7(0.9-8.3)$ & 0.082 & $0.09(0.1-4.1)$ \\
\hline Septicemia & $2.4(0.9-6.7)$ & 0.092 & $2.7(0.7-11.0)$ \\
\hline lleo & $1.2(0.7-2.1)$ & 0.446 & 0.452 \\
\hline Re-operation & $2.3(1.3-4.1)$ & 0.005 & 0.1572 \\
\hline Mortality & $1.9(1.2-3.3)$ & 0.009 & $1.02(0.3-3.9)$ \\
\hline
\end{tabular}

${ }^{*}$ mean differences in ml.. RBC : Red Blood Transfusion; OR: Odds Ratio; Cl: Confident Interval

Aspirin is not an independent factor for higher risk of postoperative complications or mortality in radical cystectomy. These data suggest that continuation of aspirin is relatively safe in this type of surgery, but larger, prospective studies are necessary to discern the true cardiovascular benefit derived from continued aspirin therapy, as well as better characterize associated bleeding risk. 